BLS 34, No 1 2008. DOI: http://dx.doi.org/10.3765/bls.v34i1.3588

(published by the Berkeley Linguistics Society and the Linguistic Society of America)

\title{
The Sentence-Internal Topic and Focus in Chinese ${ }^{1}$
}

\author{
YU-YIN HSU \\ Indiana University, Bloomington
}

\section{Introduction}

In Chinese, the object is allowed to occur in the domain between the subject and the verb, which I refer as the "sentence-internal domain," approximately below TP and above vP. This is called the preposed object construction, as in (1).

\section{(1) Zhangsan na.ben.shu ${ }_{1}$ kanguo.le $\mathrm{ec}_{1}$ Zhangsan that.CL.book read.PERF \\ 'Zhangsan has read that book.'}

In the literature, there is a dispute over the preposed object construction. Some linguists argue for such sentences as involving a Secondary Topic, as opposed to the sentence-initial Topic (e.g. Tsao 1990 and Paul 2002). Conversely, some linguists refer to such sentences as involving Focus, based on the contrastive interpretation conveyed by the sentence-internal element (e.g. Tsai 1994, Ernst and Wang 1995, Shyu 1995, and Huang 2009).

In this paper, I argue for the sentence-internal domain in Chinese. I will show that the preposed object can be either Topic or Focus in the sentence-internal domain. I will argue that two distinct projections are needed sentence internally for Topic and Focus, and that an analysis of single-projection as proposed by Lambova (2004) for Bulgarian is not supported by Chinese data. The discussion will then proceed to show how the proposed analysis accounts for the preposed-object and the so-called verb-copying constructions in Chinese.

\section{The Sentence-Internal Domain in Relation to Information Structure}

In Chinese, Topic and Focus show different syntactic properties in answering wh-questions, in the structure of emphatic shi and in terms of indefinite noun

\footnotetext{
${ }^{1}$ I am especially grateful to Dr. Yoshihisa Kitagawa for discussions and comments which have been of great help and inspiration, and I want to thank Dr. Steven Franks for his suggestions and for his always being supportive. I also thank Dr. Daniel Büring and the audience of BLS34 for their insightful comments.
} 


\section{Yu-Yin Hsu}

phrases. Assuming Chinese can license Topic and Focus in the domain of CP, the following discussion will show that the same contrast between Topic and Focus also carries over to the sentence-internal domain.

Generally, Topic can be either overt or covert in answering questions, but answers to $w h$-questions are Focus and cannot be optional. This observation holds in the sentence-internal domain of sentences in Chinese. (2a) and (2b) show that the sentence-internal item zuoye 'assignment' can be optional when it is mentioned in the previous discourse, i.e., Topic (also see Paul 2002).

$$
\begin{aligned}
& \text { ni zuoye xiewan.le ma? } \\
& \text { you assignmentwrite.PERF Q.PART } \\
& \text { 'Are you done with your assignment?' }
\end{aligned}
$$

a. wo zuoye xiewan le

I assignmentwrite PERF

'I am done with the assignment.'

$\begin{array}{clc}\text { b. wo ec } & \begin{array}{l}\text { xiewan } \\ \text { I }\end{array} & \text { le } \\ \text { write } & \text { PERF }\end{array}$

'I am done with [it].'

On the other hand, while sentence (2a) is used to answer a wh-question like (3a), the sentence-internal element zuoye 'assignment' is obligatory, since it is the answer to the wh-question. In other words, the same domain now is relevant to Focus. Furthermore, the stressed intonation on zuoye 'assignment' in (3b) indicates its Focus status.

$$
\begin{gathered}
\text { a. ni shemo xiewan.le? } \\
\text { you what write.PERF } \\
\text { 'What have you finished?' }
\end{gathered}
$$

b. wo zuoye xiewan le (zuoye can be stressed)

$$
\text { I assignmentwrite PERF }
$$

'THE ASSIGNMENT, I have finished it.'

Examples (2) and (3) suggest that the sentence-internal domain can be used for both Topic and Focus. The distinction between sentence-internal Topic and sentence-internal Focus is further supported when we look at examples of emphatic shi. In Chinese cleft sentences, emphatic shi marks Focus phrases sentence-internally. It is known that emphatic shi can occur sentence-internally to mark focused phrases.

(4) a. wo [shi zuotian] huilai de (bushi jintian)

I SHI yesterday return DE (not today)

'It is yesterday that I came back (, not today).' 


\section{b. wo [shi kanwanshu.le] \\ I SHI read.book.PERF \\ 'I did finish the reading.'}

We find that when the preposed object involves given information, it is not compatible with emphatic shi, as in (5).
a. ni zuoye xiwan.le ma?
you homework write.PERF Q-PART
'Did you do the homework?'
b.wo (*shi) zuoye xiewan.le
I SHI homework write.PERF
'I did the homework.'

However, when the preposed object is the answer to wh-questions, as in (6b), it is compatible with the emphatic shi.

$$
\begin{aligned}
& \text { (6) a. ni shemo xiwan.le? (Baogao?) } \\
& \text { you what write.PERF } \\
& \text { 'What did you finish? (Paper?)' } \\
& \text { b. wo shi zuoye xo xiewan.le (baogao hai.mei) } \\
& \text { I SHI assignment write.PERF paper not.yet } \\
& \text { 'It is the assignment that I finished (, not the paper).' }
\end{aligned}
$$

Thus, the contrast shown in (5) and (6) indicates the difference between Topic and Focus in the sentence-internal domain. The same distinction can also be found in sentences with indefinite phrases. It is pointed out by Li and Thompson (1981) that Topic in Chinese must be either generic or definite, but an indefinite Topic is not allowed. However, as reported by Tsai (1994), an indefinite phrase is allowed in the sentence-internal domain, e.g. yi.pian.lunwen 'one paper' in (7).

(7) wo yi.pian.lunwen keyi yingfu (, liang.pian jiu bu xing le )

$$
\begin{aligned}
& \text { I one.CL.paper can handle } 2 \text { 2. CL.paper then not can ASP } \\
& \text { 'I can handle ONE PAPER (, but not two).' }
\end{aligned}
$$

Example (7) suggests that besides Topic, the sentence-internal position can host a Focus phrase. Given the fact that emphatic shi is compatible only with Focus but not with Topic, we can see in example (8) that the sentence-internal indefinite NP in (7), yi.pian.lunwen 'one paper', is compatible with emphatic shi as expected.
(8) wo shi yi.pian.lunwen keyi yingfu
(, liang.pian jiu bu xing le)
I SHI 1.CL.paper can handle
2. CL.paper then not can ASP
'It is one paper that I can handle (, but not two).'


Yu-Yin Hsu

The previous discussion shows that both Topic and Focus are available to NPs in the sentence-internal domain in Chinese, and that such Topic and Focus do show different properties syntactically. It then further suggests that treating the sentence-internal noun phrase as either Topic (e.g., Tsao 1990 and Paul 2002) or Focus (e.g., Tsai 1994 and Shyu 1995) only illustrates part of the facts. Therefore, in the spirit of Rizzi (1997), I propose that two functional projections, TopP and FocP, are available in the sentence-internal domain to host Topic and Focus when needed.

\section{Proposal: TopP and FocP in the Sentence-Internal Domain}

The previous section shows that the preposed object involves either Topic or Focus interpretation. To account for this fact, I claim that two distinct projections should be identified in the sentence-internal domain (i.e., between TP and $v \mathrm{P}$ ) to host Topic and Focus respectively. The proposed structure is shown in (9), where TopP and FocP sit between TP and $v$, and TopP is higher than FocP.

(9) TopP and FocP in the sentence-internal domain

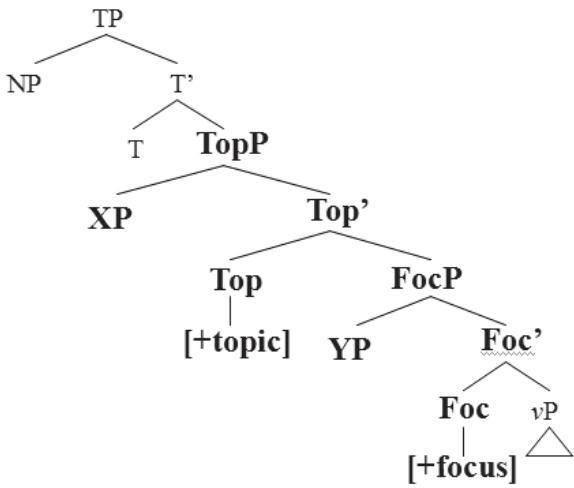

The gist of the proposed structure is that, in the sentence-internal domain, both Topic and Focus can be licensed when proper contexts are provided. This structure predicts that Topic and Focus can co-occur in the sentence-internal domain, with Topic always higher than Focus. The prediction is borne out. In (10a), Topic shu 'book' and Focus xiaoshuo 'novel' co-occur in the sentence-internal domain, and they have to occur in a fixed order (e.g. (10b)).

(10) 'Speaking of books, it is novels that he reads most.'
a. ta
shu-TOP
xiaoshuo-Foc kan.de
zui duo
he book novel
read.RESULT
mostmany
b. *ta xiaoshuo-FOC
shu-TOP kan.de
zui duo
he book
book
read.RESULT
mostmany. 


\section{The Sentence-internal Topic and Focus in Chinese}

Moreover, the following examples show when there are two elements in the sentence-internal domain, the differences between Topic and Focus still holds. With respect to $w h$-questions, the discourse in (11) shows that the Topic NP in the sentence-internal domain (i.e. shuiguo 'fruit') is optional, but the focus NP is obligatory (i.e. pingguo 'apple').

a. ta shuiguo shemo zui.chang chi?
he fruit what most.often eat
'Speaking of fruit, what does he eat most often?'
b. ta (shuiguo) *(pingguo) zui.chang chi
he fruit apple most often eat
(Fruit,) he eats apples most often.'

The contrast between Topic and Focus in the sentence-internal domain is further supported by cleft sentences. In (12), emphatic shi is only compatible with Focus NP xiaoshuo 'novel', as shown in the contrast between (12a) and (12b).

(12) 'Speaking of books, it is novels that he reads most.'
a. *ta [shi shu $]$ xiaoshuo kan.de zui.duo
he SHI book novel read.DE most.many
b. ta shu [shi xiaoshuo] kan.de zui.duo
he book SHI novel read.DE most.many

Different analyses of emphatic shi have been proposed in the literature. Chiu (1993) proposes that emphatic shi heads a functional projection as ShiP. Lee (2005) argues that emphatic shi is a grammaticalized focus marker heads a focus phrase. Based on facts of ellipsis, Li in progress argues that emphatic shi is subcategorized for an IP. It has also been argued that emphatic shi is generated in the split INFL domain and projects its functional projection, as proposed by Huang $(1988,2009)$. I thereby adopt the approach that emphatic shi is analyzed as heading its functional projection. Its intervention indicates that Topic and Focus in the sentence-internal domain do not form a constituent (contra Lambova (2004). The proposed structure is thus supported. It is then predicted that sentences with a ditransitive verb like song 'send' would allow both direct and indirect objects to be preposed in the sentence-internal domain. ${ }^{2}$ The prediction is borne out as shown in example (13). Unlike the canonical pattern as in (13a), sentences like (13b) and (13c) convey interpretations for specific pragmatic contexts.

(13) a. wo zuotian song.le Zhangsan na.ben.Chomsky.de ${ }^{3}$ shu I yesterday send.PERF Zhangsan that.CL.Chomsky.DE book 'Yesterday, I sent Zhangsan that book of Chomsky'

\footnotetext{
2 Thanks to Dr. Daniel Büring for pointing this out to me.

3 The marker $-d e$ is for nominal modifiers.
} 
Yu-Yin Hsu

b. wo, Zhangsan, na.ben.Chomsky.de shu zuotian song.le I Zhangsan that.CL.Chomsky.DE book yesterday send.PERF 'Speaking of Zhangsan, I sent [him] THAT BOOK OF CHOMSKY.'

c. wo, na.ben.Chomsky.de shu, Zhangsan song.le (qita.ren haimei) I that.CL.Chomsky.DEbook Zhangsan send.PERF other.person not.yet 'As for that book of Chomsky, it is to Zhangsan that I sent it already (, but not to other people).'

In sum, I agree with the previous analyses of the preposed object as proposed by Tsai (1995) and Shyu (1995) for Focus and by Paul (2002) for Topic, but depart from them in arguing that both Topic and Focus are allowed to license the preposed object in Chinese (cf. Belletti 2004 for Topic and Focus in the low IP area in Italian). Given the proposed structure, in the following section, I will show that the so-called "verb-copying construction" can be accounted for by the present analysis.

\section{The So-Called Verb-Copying Construction}

Since Huang (1982), it has been noticed that Chinese has a construction referred to as like Verb-copying (or "verb duplication" in Huang 1982) construction. In Chinese, a transitive verb cannot be followed by an object together with a complement phrase denoting the duration (e.g., 14a) or by a resultative phrase (e.g., 14b). In other words, there is at most one complement allowed for each verb.
(14) a. * ta kan.le [shu] [liang.ge.xiaoshi]
he read.PERF book 2.CL.hour
'He has read books for two hours.'
b. *ta kan.le [shu] [hen lei]
he read.PERF book very tired
'He read books and got very tired.'

Therefore, "an extra copy of the head verb" is needed to salvage sentences like (14), as shown in (15).

(15) a. ta [kan shu] [kan.le liang.ge xiaoshi]

he read book read.PERF 2.CL.hour

'He has read books for two hours.

b. ta [kan shu] [kan.de hen lei]

he read book read.RESULT very tired

'He read books and got very tired.'

In terms of the construction at issue, Huang argues for a VP adjunction analysis, with the structure is in (16). He claims that such duplication is motivated 


\section{The Sentence-internal Topic and Focus in Chinese}

by a PF filter, viz. copying the verb for extra complement, and then the original VP1 functions like an adverbial of the duplicated V2 in forming a bigger VP unit. ${ }^{4}$
(16) ta $[\mathrm{vp}[\mathrm{v} 1$, qi -original
he ride
ma ] [v2 qi -duplicated.le $_{\text {. }}$
xan.ge.xiaoshi]]
'He rode for three hours.'
horse ride PERF
3.CL.hour

However, there are some problems shared by VP-analyses. For example, the relative position of the $b a$-phrase is problematic for mono-clausal VP analyses, because it is not clear why VP1 never follows $b a$-phrases, if VP1 and its following VP form a bigger VP and $b a$-phrases indicate the left-periphery of VP. (17) shows that the $b a$-phrase occurs between VP1 and V2, but it cannot precede VP1.
(17) ta (*ba ma) [vp1xunlian 1 ma] (ba ma) xunlian 2 .de hen hao he BA horse train horse BAhorse train.RESULT very well 'He trains horses very well.'

This suggests that the VP1 xunlian ma 'train horse' in (17) is at a position outside of the predicate-VP. Besides, if VP1 is analyzed as part of the predicate-VP, it is not clear why the distribution of VP1 is similar to that of temporal or locatives expressions. As in (18a), VP1 is a constituent independent of the predicate-VP.

\section{(18) a. ([vp1 kan shu]) ta ([vp1 kan shu]) keyi ([vp1 kan shu]) kan ${ }_{2}$ haojige.xiaoshi} readbook he readbook can readbook read many.hour

'he can read books for many hours.'

b. (jintian/zai.zheli) wo (jintian/zai.zheli) keyi (jintian/zai.zheli) kan shu today/at home I today/at home can today/at home read book 'Today/At home, I can read books.'

Also, under VP-analyses, it is not clear why the "duplicated" V2 can carry aspect markers but the "original" VP1 never does. In (19), when V1 takes aspect markers, the sentences become ungrammatical (i.e. (19b) and (19c)).

\footnotetext{
${ }^{4}$ To simplify the discussion, I refer to the original verb and its complement as VP1 and the duplicated verb as V2.
} 
Yu-Yin Hsu

(19) 'He read books for three hours.'
a. ta $\mathrm{kan}_{1}$ shu kan2.le
san.ge.xiaoshi
he read book read.PERF
3.CL.hour
b. *ta $\operatorname{kan}_{1}$. le
shu $\mathrm{kan}_{2}$ san.ge.xiaoshi
he read.PERF
book read 3.CL.hour
c. *ta $\operatorname{kan}_{1} .1 \mathrm{e}$
shu $\mathrm{kan}_{2} . \mathrm{le} \quad$ san.ge.xiaoshi
he read.PERF
book read.PERF 3.CL.hour

Therefore, unlike VP-analyses, I argue that the so-called verb-copying construction in Chinese is better accounted for under the present analysis. I propose that the VP1 at issue can be base-generated at TopP or FocP in the sentence-internal domain, where it gets the corresponding interpretation. The realization of the sentence-internal VP in each functional projection is discussed as follows.

As shown in structure (20), VP1 can be base-generated as Topic in the sentence-internal domain, while VP2 is the predicate of the sentence.

(20) VP1 as Topic in the sentence-internal domain

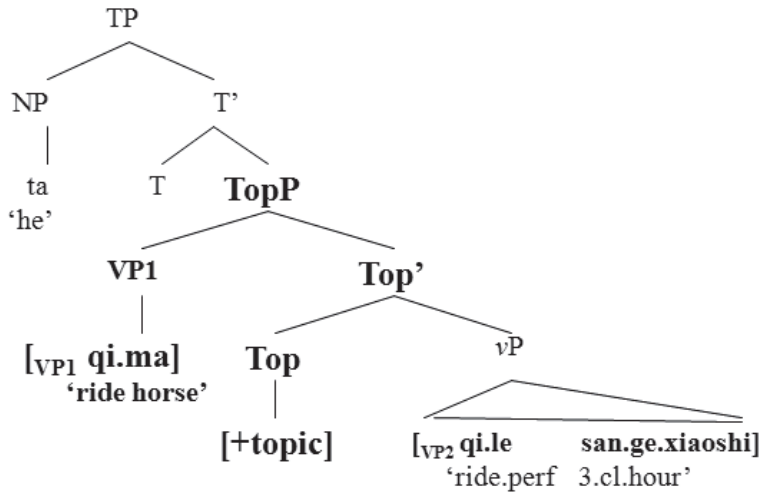

In a discourse like (21), VP1 xue zhongwen 'learn Chinese' in (21b) is optional in answering (21a), because it is mentioned in question (21a), hence is the Topic.

(21) a. ni [vp1xue zhongwen] xue 2. le wu.nian ma? you learn Chinese learn.PERF five.year Q.PART 'Did you learn Chinese for 5 years?'

b. dui, wu ([vp1xue zhongwen]) xue $2.1 \mathrm{l}$ wu.nian yes, I learn Chinese learn.PERF 5.year 'Yes, I spent five years learning Chinese.' 
The other supporting evidence comes from lian-phrases. Lian-phrases are analyzed as Focus phrases with the marker lian- 'even' in Chinese (see Shyu 1995 and Paul 2002). We find that when a lian-phrase occurs in the sentence-internal domain, VP1 is most naturally interpreted as Topic and VP1 must precede the lian-phrase:

(22) ta (*lian minima) [vp1 qi ma] lian minima dou qi $i_{2}$ bu hao he even mini.horse ride horse even mini.horse all ride not.well 'He can't ride well, even a mini.horse.'

Therefore, I argue that VP1 can be licensed as Topic in the sentence-internal domain. It follows that such Top-VP1 cannot take aspect markers (e.g. (19)) and that it has a freer distribution, since Chinese also allows Topic in the CP domain (e.g. (18)). Moreover, it comes as a natural result that $b a$-phrases cannot precede Top-VP1 (e.g. (17)). The present analysis also predicts that VP1 has to precede VP2, since Top-VP1 is higher than the predicate-VP. Examples that are problematic to VP analyses are thus explained.

Given the proposed two-projection analysis, one may wonder about another possible location for VP1, i.e., the Spec-Foc. The corresponding structure is shown in (23).

(23) VP1 as Focus in the sentence-internal domain

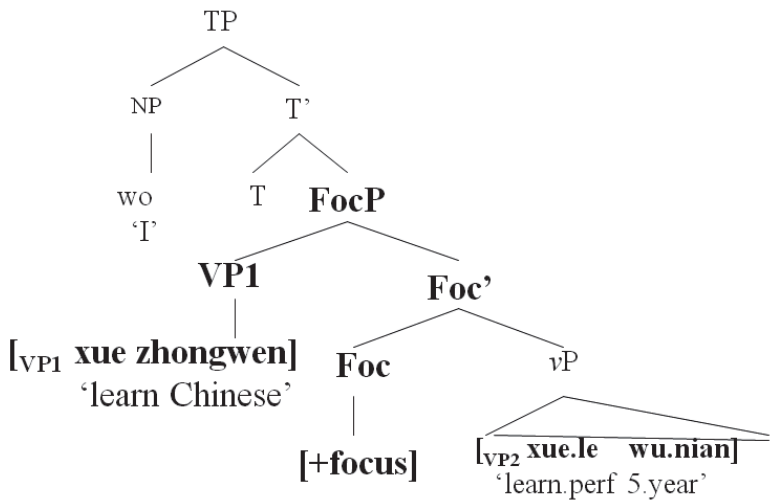

The Focus status of VP1 is shown when by the answers to wh-questions. We see that VP1 is used to answer the question in (24a), which suggests that VP1 in (24b) xue zhongwen 'learn Chinese' is Focus in the sentence-internal domain.
(24) a. ni
shemo xue.le
wu.nian?
you what learn.PERF 5.year
'What have you learned for five years?' 


\title{
Yu-Yin Hsu
}

\author{
b. wo [vp1 xue zhongwen] [vP2Xue.le wu.nian] \\ I learn Chinese learn.PERF 5.year \\ 'I have learned Chinese for five years.'
}

The preceding discussion shows that the sentence-internal domain can license base-generated VPs as Topic or Focus. The proposed structure predicts that the co-occurrence of internal Topic and internal Focus should be allowed. The sentence in (25) shows two VPs occurring before the predicate and after the subject of the sentence, where the former zuo yundong 'do exercise' receives the Topic interpretation and the latter da wangqui 'play tennis', the Focus.

(25) ta [Top-VP zuo yundong] [Foc-VP da wangqui] keyi [vp [vp da haoji.xiaoshi]] he do exercise play tennis can play many.hour 'Speaking of doing exercise, it is playing tennis that he can do for a long time.'

It is thereby confirmed that Topic and Focus can be licensed in the sentence-internal domain in Chinese and that the so-called verb-copying construction in fact involves base-generated VPs as Topic/Focus.

Following this line of analysis, there are examples which further support the proposed analysis. The relative order of sentential adverbs and modals suggests that VP1 is licensed at the INFL domain, higher than the predicate-VP.

(26) ta (xianran) qi.ma (xianran) qi.de (*xianran) hen.lei he obviously ride.horse obviously ride.RESULT obviously very.tired 'Obviously, he got very tired because of riding.'

(27) ta (yinggai) kan.shu (yinggai) kan.le (*yinggai) shi.ge.xiaoshi he should see.book should see.PERF should ten.CL.hour 'He should have read [a book] for 10 hours.'

Sentences like (26) and (27) are expected given the present analysis with distinct functional projections. In addition, sentences with emphatic shi further support the distinction between Top-VP and Foc-VP in the sentence-internal domain. As shown by (28a), when VP1 xue zhongwen 'learn Chinese' is the Focus of the sentence, it is compatible with the emphatic shi. However, in (28b), the same VP1 with a Topic interpretation is not compatible with the emphatic shi.

(28) a. Focus

wo shi xue.zhongwen xue.le wu nian (, bu shi xue hanyu)

I SHI learn.Chinese learn.PERF 5.year

'It is learning Chinese that I spent five years doing (, not learning Korean).' 
b. Topic

*wo shi xue.zhongwen xue.le wu nian (, bu shi si nian)

I SHI learn.Chinese learn.PERF 5.year

'Speaking of learning Chinese, it is five years that I spent doing it (not four years).'

Furthermore, sentences with co-occurring VPs are also possible. Example (29) shows that VP licensed in TopP (e.g., zuo.yundong 'do.exercise'), is not compatible with emphatic shi and that a following VP licensed in FocP (e.g., da.wangqui 'play tennis') is fine with the emphatic shi. Thus, it is concluded that VPs in the sentence-internal domain should be analyzed as being in the projection of TopP or FocP, rather than as part of the predicate VP.

(29) ta (*shi) [Top-Vp Zuo yundong] (shi) [Foc-VP da wangqui] keyi he SHI do exercise SHI playtennis can [vP [vP da haoji.xiaoshi]]

play many hour

'Speaking of doing exercise, it is playing tennis that he can do for a long time.'

\section{Concluding Remarks}

Given that Chinese is an SVO language, it has been a point of dispute whether the object preposed to the position between the subject and the predicate is a Topic (e.g., Tsao 1990 and Paul 2002) or a Focus (e.g., Tsai 1994 and Shyu 1995). Through the careful examination of this and other constructions, this paper argued that the "split-CP" approach à la Rizzi (1997) can and should be extended to the sentence-internal domain in Chinese, enabling Topic and/or Focus to appear, which echoes claims in Belletti (2004) about Italian syntax, but contrary to Lambova's (2004) analysis of single projection. The present study also showed that the phenomenon of the preposed object is better accounted for under such an analysis. In other words, both Topic and Focus are available to NPs in the sentence-internal domain when proper contexts are provided. All these facts can be captured by postulating Topic and Focus projections in this hierarchical order in the sentence-internal domain, i.e., between TP and $v \mathrm{P}$, on a par with the CP domain. This analysis can account for the information structure carried by the sentence-internal elements, their co-occurrence and their ordering restriction. The proposed analysis also applies to the so-called verb-copying construction in Chinese. By this joint approach of syntax and the information structure, I demonstrated that issues related to the so-called verb-copying sentences, such as fixed ordering and aspect-marker taking, are properly accounted for, and that problems in the previous analyses are avoided. Finally, the preposed object construction and the so-called verb-copying construction can be accounted for by a unified account. 
Yu-Yin Hsu

\section{References}

Belletti, Adriana. 2004. Aspects of the Low IP Area. In The Structure of CP and IP. Luigi Rizzi (ed.) Pp 16-51.

Chiu, Bonnie Hui-chun. 1993. The Inflectional Structure of Chinese. Ph.D. diss., University of California, Los Angeles.

Ernst, Tomas and Chengchi Wang. 1995. Object Preposing in Mandarin Chinese. Journal of East Asian Linguistics 4:3, 235-260.

Huang, C.-T. James. 1982. Logical Relations in Chinese and the Theory of Grammar. New York \& London: Garland Publishing, Inc.

Huang, C.-T. James. 1988. On 'Be' and 'Have' in Chinese (Shuo Shi gen You). The Bulletin of the Institute of History and Philosophy Vol. LIX, Part I. Taipei: Academica Sinica.

Huang, C.-T. James., Audrey Li and Yafei Li. 2009. The Syntax of Chinese. Cambridge University Press.

Lambova, Mariana Dobreva. 2004. On Information Structure and Clausal Architecture: Evidence from Bulgarian. Ph.D. diss., University of Connecticut.

Lee, Hui-Chi. 2005. On Chinese Focus and Cleft Constructions. Ph.D. diss., National Tsing Hua University, Taiwan.

Li, Charles N. and Sandra A. Thompson. 1981. Mandarin Chinese: A Functional Reference Grammar. Berkeley: University of California Press.

Paul, Waltraud. 2002. Sentence-internal Topics in Mandarin Chinese: The Case of Object Preposing. Language and Linguistics 3:4, 695-714.

Rizzi, Luigi. 1997. The Fine Structure of the Left Periphery. In L. Haegeman, ed., Elements of Grammar: Handbook in Generative Syntax, 281-337. Dordrecht: Kluwer Academic Publishers.

Shyu, Shu.-Ing. 1995. The Syntax of Focus and Topic in Mandarin Chinese. University of Southern California: Dissertation.

Tsai, Wei.-Tian. Dylan. 1994. On Economizing the Theory of $A^{\prime}$ Dependencies. Ph.D. diss., Massachusetts Institute of Technology.

Tsao, Feng.-Fu. 1990. Sentence and Clause Structure in Chinese: A Functional Perspective. Taipei: Student Book, Co.

Yu-Yin Hsu

Indiana University, Bloomington

Department of Linguistics; Memorial Hall 322

1021 East Third Street

Bloomington, IN, 47405-7005

hsuy@indiana.edu 